\title{
Affine deformations of a three-holed sphere
}

\author{
VIRGINIE CHARETTE \\ TODD A DRUMM \\ WILLIAM M GOLDMAN
}

\begin{abstract}
Associated to every complete affine 3-manifold $M$ with nonsolvable fundamental group is a noncompact hyperbolic surface $\Sigma$. We classify these complete affine structures when $\Sigma$ is homeomorphic to a three-holed sphere. In particular, for every such complete hyperbolic surface $\Sigma$, the deformation space identifies with two opposite octants in $\mathbb{R}^{3}$. Furthermore every $M$ admits a fundamental polyhedron bounded by crooked planes. Therefore $M$ is homeomorphic to an open solid handlebody of genus two. As an explicit application of this theory, we construct proper affine deformations of an arithmetic Fuchsian group inside $\operatorname{Sp}(4, \mathbb{Z})$.
\end{abstract}

57M05; 20H10, 30F60

\section{Introduction}

A complete affine manifold is a quotient

$$
M=\mathbf{E} / \Gamma
$$

where $\mathbf{E}$ is an affine space and $\Gamma \subset \operatorname{Aff}(\mathbf{E})$ is a discrete group of affine transformations of $\mathbf{E}$ acting properly and freely on $\mathbf{E}$. When $\operatorname{dim} \mathbf{E}=3$, Fried-Goldman [19] and Mess [29] imply that either

- $\Gamma$ is solvable, or

- $\Gamma$ is virtually free.

When $\Gamma$ is solvable, $M$ admits a finite covering homeomorphic to the total space of a fibration composed of cells, circles, annuli and tori. The classification of structures in this case is straightforward [19]. When $\Gamma$ is virtually free, the classification is considerably more interesting. In the early 1980's Margulis [27; 28] discovered the existence of such structures, answering a question posed by Milnor [30].

Tameness Conjecture Suppose $M^{3}$ is a 3-dimensional complete affine manifold with free fundamental group. Then $M$ is homeomorphic to an open solid handlebody. 
This is the analog of Marden's Tameness Conjecture, recently proved by Agol [2] and Calegari-Gabai [4].

By Fried-Goldman [19], the linear holonomy homomorphism

$$
\operatorname{Aff}\left(\mathbf{E}^{3}\right) \stackrel{\mathrm{L}}{\longrightarrow} \mathrm{GL}(3, \mathbb{R})
$$

embeds $\Gamma$ as a discrete subgroup of $\mathrm{GL}(3, \mathbb{R})$ conjugate to the orthogonal group $\mathrm{O}(2,1)$. Thus $M$ admits a complete flat Lorentz metric and is a (geodesically) complete flat Lorentz 3-manifold. We henceforth restrict our attention to the case when $\mathbf{E}$ is a 3-dimensional Lorentzian affine space $\mathbf{E}_{1}^{3}$. A Lorentzian affine space is a simply connected geodesically complete flat Lorentz 3-manifold, and is unique up to isometry.

Furthermore $L(\Gamma)$ is a Fuchsian group acting properly and freely on the hyperbolic plane $\mathbf{H}^{2}$. We model $\mathbf{H}^{2}$ on a component of the two-sheeted hyperboloid

$$
\left\{v \in \mathbb{R}_{1}^{3} \mid v \cdot v=-1\right\},
$$

or equivalently its projectivization in $\mathrm{P}\left(\mathbb{R}_{1}^{3}\right)$. (Compare Goldman [21].) The quotient

$$
\Sigma:=\mathbf{H}^{2} / \mathrm{L}(\Gamma)
$$

is a complete hyperbolic surface homotopy-equivalent to $M$, naturally associated to the Lorentz manifold $M$.

We prove the Tameness Conjecture in the first nontrivial case, that is, when the surface $\Sigma$ is homeomorphic to a three-holed sphere.

Let $\Gamma_{0} \subset \mathrm{O}(2,1)$ be a Fuchsian group. Denote the corresponding embedding

$$
\rho_{0}: \Gamma_{0} \hookrightarrow \mathrm{O}(2,1) \subset \mathrm{GL}(3, \mathbb{R}) .
$$

Let $G \subset \operatorname{Aff}\left(\mathbf{E}_{1}^{3}\right)$ denote the group of affine isometries of $\mathbf{E}_{1}^{3}$. An affine deformation of $\Gamma_{0}$ is a representation

$$
\Gamma_{0} \stackrel{\rho}{\rightarrow} \mathrm{G}
$$

satisfying $L \circ \rho=\rho_{0}$. We refer to the image $\Gamma$ of $\rho$ as an affine deformation as well. An affine deformation is proper if the affine action of $\Gamma_{0}$ on $\mathbf{E}_{1}^{3}$ defined by $\rho$ is a proper action.

Margulis [27; 28] discovered proper actions by bounding from below the Euclidean distance that elements of $\Gamma$ displace points. Our more geometric approach constructs fundamental polyhedra for affine deformations in the spirit of Poincaré's theorem on fundamental polyhedra for hyperbolic manifolds. 
This approach began with Drumm [14], who constructed fundamental polyhedra from crooked planes to show that certain affine deformations $\Gamma$ act properly on all of $\mathbf{E}_{1}^{3}$. A crooked plane is a polyhedron in $\mathbf{E}_{1}^{3}$ with four infinite faces, adapted to the invariant Lorentzian geometry of $\mathbf{E}_{1}^{3}$. Specifically, representing the hyperbolic surface $\Sigma$ as an identification space of a fundamental polygon for the generalized Schottky group $\mathrm{L}(\Gamma) \subset \mathrm{O}(2,1)$, we construct a fundamental polyhedron for certain affine deformations $\Gamma$ bounded by crooked planes [14]. We call such a fundamental polyhedron a crooked fundamental polyhedron.

Crooked Plane Conjecture Suppose $\Gamma \subset \mathrm{G}$ is a discrete group acting properly on $\mathbf{E}_{1}^{3}$. Suppose $\Gamma$ is not solvable. Then some finite-index subgroup of $\Gamma$ admits a crooked fundamental polyhedron.

Clearly an affine deformation $\Gamma$ for which a finite-index subgroup admits a crooked fundamental polyhedron is proper, and the quotient is an open solid handlebody. Thus the Crooked Plane Conjecture implies the Tameness Conjecture. We prove the Crooked Plane Conjecture when $\Sigma$ is homeomorphic to a three-holed sphere.

Theorem (Drumm) Every free discrete Fuchsian group $\Gamma_{0} \subset \mathrm{O}(2,1)$ admits a proper affine deformation.

Actions of free groups by Lorentz isometries are the only cases to consider. FriedGoldman [19] reduces the problem to when $\Gamma_{0}$ is a Fuchsian group, and Mess [29] implies $\Gamma_{0}$ cannot be cocompact. (See Goldman-Margulis [23] and Labourie [26] for alternate proofs.) Thus, after passing to a finite-index subgroup, we may assume that $\Gamma_{0}$ is free.

The linear representation $\rho_{0}$ is itself an affine deformation, by composing it with the embedding

$$
\mathrm{GL}(3, \mathbb{R}) \hookrightarrow \operatorname{Aff}\left(\mathbf{E}_{1}^{3}\right) .
$$

Slightly abusing notation, denote this composition by $\rho_{0}$ as well. Two affine deformations are translationally equivalent if they are conjugate by a translation in $\mathbf{E}_{1}^{3}$. An affine deformation is trivial (or radiant) if and only if it is translationally equivalent to the affine deformation $\rho_{0}$ constructed above. In other words an affine deformation is trivial if it fixes a point in the affine space $\mathbf{E}_{1}^{3}$.

Let $\mathbb{R}_{1}^{3}$ denote the vector space underlying the affine space $\mathbf{E}_{1}^{3}$, considered as a $\Gamma_{0}-$ module via the linear representation $\rho_{0}$. The space of translational equivalence classes of affine deformations of $\rho_{0}$ identifies with the cohomology group $\mathrm{H}^{1}\left(\Gamma_{0}, \mathbb{R}_{1}^{3}\right)$. For 
each $g \in \Gamma_{0}$, define the translational part $u(g)$ of $\rho(g)$, as the unique translation taking the origin to its image under $\rho(g)$. That is, $u(g)=\rho(g)(0)$, and

$$
x \stackrel{\rho(g)}{\longmapsto} \rho_{0}(g)(x)+u(g) .
$$

The map $\Gamma_{0} \stackrel{u}{\rightarrow} \mathbb{R}_{1}^{3}$ is a cocycle in $Z^{1}\left(\Gamma_{0}, \mathbb{R}_{1}^{3}\right)$, and conjugating $\rho$ by a translation changes $u$ by adding a coboundary.

The classification of complete affine structures in dimension 3 therefore reduces to determining, for a given free Fuchsian group $\Gamma_{0}$, the subset of $\mathrm{H}^{1}\left(\Gamma_{0}, \mathbb{R}_{1}^{3}\right)$ corresponding to translational equivalence classes of proper affine deformations.

Margulis [27; 28] introduced an invariant of the affine deformation $\Gamma$, defined for elements $\gamma \in \Gamma$ whose linear part $\mathrm{L}(\gamma)$ is hyperbolic. Namely, $\gamma$ preserves a unique affine line $C_{\gamma}$ upon which it acts by translation. Furthermore $C_{\gamma}$ inherits a canonical orientation. As $C_{\gamma}$ is spacelike, the Lorentz metric and the canonical orientation determines a unique orientation-preserving isometry

$$
\mathbb{R} \stackrel{j_{\gamma}}{\longrightarrow} C_{\gamma}
$$

The Margulis invariant $\alpha(\gamma) \in \mathbb{R}$ is the displacement of the translation $\left.\gamma\right|_{C_{\gamma}}$ as measured by $j_{\gamma}$ :

$$
j_{\gamma}(t) \stackrel{\gamma}{\longmapsto} j_{\gamma}(t+\alpha(\gamma))
$$

for $t \in \mathbb{R}$.

Margulis's invariant $\alpha$ is a class function on $\Gamma_{0}$ which completely determines the translational equivalence class of the affine deformation (see Drumm-Goldman [18] and Charette-Drumm [9]). Charette-Drumm [8] extended Margulis's invariant to parabolic transformations. However, only its sign is well defined for parabolic transformations. To obtain a precise numerical value one requires a decoration of $\Gamma_{0}$, that is, a choice of horocycle at each cusp of $\Sigma$.

If $\Gamma$ is an affine deformation of $\Gamma_{0}$ with translational part $u \in Z^{1}\left(\Gamma_{0}, \mathbb{R}_{1}^{3}\right)$, then we indicate the dependence of $\alpha$ on the cohomology class $[u] \in \mathrm{H}^{1}\left(\Gamma_{0}, \mathbb{R}_{1}^{3}\right)$ by writing $\alpha=\alpha_{[u]}$.

Let $\Gamma_{0}$ be a Fuchsian group whose corresponding hyperbolic surface $\Sigma$ is homeomorphic to a three-holed sphere. Denote the generators of $\Gamma_{0}$ corresponding to the three ends of $\partial \Sigma$ by $g_{1}, g_{2}, g_{3}$. Choose a decoration so that the generalized Margulis 
invariant defines an isomorphism

$$
\begin{aligned}
\mathrm{H}^{1}\left(\Gamma_{0}, \mathbb{R}_{1}^{3}\right) & \longrightarrow \mathbb{R}^{3} \\
{[u] } & \longmapsto\left[\begin{array}{l}
\mu_{1}([u]) \\
\mu_{2}([u]) \\
\mu_{3}([u])
\end{array}\right]:=\left[\begin{array}{l}
\alpha_{[u]}\left(g_{1}\right) \\
\alpha_{[u]}\left(g_{2}\right) \\
\alpha_{[u]}\left(g_{3}\right)
\end{array}\right] .
\end{aligned}
$$

Theorem A Let $\Gamma_{0}, \Sigma, \mu_{1}, \mu_{2}, \mu_{3}$ be as above. Then $[u] \in \mathrm{H}^{1}\left(\Gamma_{0}, \mathbb{R}_{1}^{3}\right)$ corresponds to a proper affine deformation if and only if

$$
\mu_{1}([u]), \mu_{2}([u]), \mu_{3}([u])
$$

all have the same sign. Furthermore in this case $\Gamma$ admits a crooked fundamental polyhedron and $M$ is homeomorphic to an open solid handlebody of genus two.

For purely hyperbolic $\Gamma_{0}$, Theorem A was proved by Jones in her doctoral thesis [25], using a different method.

In the case that $\Sigma$ is a three-holed sphere, Theorem A gives a complete description of the deformation space and the topological type. As three-holed spheres are the building blocks of hyperbolic surfaces, the present paper plays a fundamental role in our investigation of affine deformations of hyperbolic surfaces of arbitrary topological type. Except in a few other conjectural cases, for example when $\Sigma$ is homeomorphic to a two-holed projective plane (or cross-surface) or one-holed Klein bottle, the situation is more complicated as the deformation space will be defined by infinitely many inequalities. When $\Sigma$ supports irrational measured geodesic laminations, as on the one-holed torus, the deformation space may be a convex domain with fractal boundary (see Goldman-Margulis-Minsky [24]).

Margulis's opposite sign lemma [27; 28] (see Abels [1] for a beautiful exposition) states that uniform positivity (or negativity) of $\alpha(\gamma)$ is necessary for properness of an affine deformation. In Goldman-Margulis [23] and Goldman [20] uniform positivity or negativity of $\alpha(\gamma)$ was conjectured to be equivalent to properness. Theorem A implies this conjecture when $\Sigma$ is a three-holed sphere with geodesic boundary. In that case only the three $\gamma$ corresponding to $\partial \Sigma$ need to be checked. However, when $\Sigma$ has at least one cusp, Theorem A provides counterexamples to the original conjecture. If the generalized Margulis invariant of that cusp is zero, and those of the other ends are positive, then $\alpha(\gamma)>0$ for all hyperbolic elements $\gamma \in \Gamma$. Other counterexamples are given in Goldman-Margulis-Minsky [24].

For some surfaces more complicated than the 3-holed sphere, positivity of $\alpha(\gamma)$ for finitely many elements $\gamma$ will be insufficient to guarantee properness of the action. (See Charette [6; 7] for specific examples.) 
We apply Theorem A to construct a proper affine deformation of an arithmetic group in $\operatorname{SL}(2, \mathbb{Z})$ inside $\operatorname{Sp}(4, \mathbb{Z})$. Here $G$ is represented as the subgroup of $\operatorname{Sp}(4, \mathbb{R})$ stabilizing the Lagrangian plane $\mathbb{R}^{2} \oplus 0$ in a symplectic vector space $\mathbb{R}^{4}$ defined over $\mathbb{Z}$. The set of Lagrangian 2-planes in $\mathbb{R}^{4}$ transverse to $\mathbb{R}^{2} \oplus 0$ is naturally a model for $\mathbf{E}_{1}^{3}$ and $\Gamma$ acts by affine Lorentz isometries. This model of Minkowski space embeds in the conformal compactification of $\mathbf{E}_{1}^{3}$, the Einstein universe (see Barbot et al [3]) upon which $\operatorname{Sp}(4, \mathbb{R})$ acts transitively.

Example For $i=1,2,3$ choose three positive integers $\mu_{1}, \mu_{2}, \mu_{3}$. Then the subgroup $\Gamma$ of $\mathrm{Sp}(4, \mathbb{Z})$ generated by

$$
\left[\begin{array}{cccc}
-1 & -2 & \mu_{1}+\mu_{2}-\mu_{3} & 0 \\
0 & -1 & 2 \mu_{1} & -\mu_{1} \\
0 & 0 & -1 & 0 \\
0 & 0 & 2 & -1
\end{array}\right],\left[\begin{array}{cccc}
-1 & 0 & -\mu_{2} & -2 \mu_{2} \\
2 & -1 & 0 & 0 \\
0 & 0 & -1 & -2 \\
0 & 0 & 0 & -1
\end{array}\right]
$$

acts properly and freely, with quotient manifold homeomorphic to a solid open handlebody of genus two.

Our result complements recent work of Goldman-Labourie-Margulis [22]. When the hyperbolic surface $\Sigma$ is convex cocompact, the space of proper affine deformations identifies with an open convex cone in $\mathrm{H}^{1}\left(\Gamma_{0}, \mathbb{R}_{1}^{3}\right)$ defined by the nonvanishing of a generalization of the Margulis invariant to geodesic currents on $\Sigma$.

This cone is the interior of the intersection of half-spaces defined by the functionals

$$
\begin{aligned}
H^{1}\left(\Gamma_{0}, \mathbb{R}_{1}^{3}\right) & \longrightarrow \mathbb{R} \\
{[u] } & \longmapsto \alpha_{[u]}(g)
\end{aligned}
$$

for $g \in \Gamma_{0}$. In general we expect this cone to be the union of open regions corresponding to combinatorial configurations realized by crooked planes, thereby giving a crooked fundamental polyhedron for each proper affine deformation. Jones [25] used standard Schottky fundamental domains to fill the open cone with such regions. In the present paper, we decompose $\Sigma$ into two ideal triangles, obtaining a single combinatorial configuration which applies to all proper affine deformations.

The presentation in this paper focuses on the case of uniformly positive Margulis invariants. While the arguments for negative invariants boil down to sign changes, including them is unnecessarily cumbersome. Occasionally, we will mention how to modify the approach in that case. 
Acknowledgements We are grateful to Ian Agol, Francis Bonahon, Dick Canary, David Gabai, Ryan Hoban, Cathy Jones, François Labourie, Misha Kapovich, Grisha Margulis, Yair Minsky, and Anna Wienhard for helpful discussions. We also wish to thank the Institute for Advanced Study for their hospitality. We thank the anonymous referee for numerous helpful suggestions.

Charette gratefully acknowledges partial support from the Natural Sciences and Engineering Research Council of Canada and from the Fonds québécois de la recherche sur la nature et les technologies. Goldman gratefully acknowledges partial support from National Science Foundation grants DMS070781 and the Oswald Veblen Fund at the Institute for Advanced Study.

\section{Lorentzian geometry}

This section summarizes needed technical background on the geometry of Minkowski $(2+1)$-spacetime, its isometries, and Margulis's invariant of hyperbolic and parabolic isometries. For details, variants, and proofs, see Abels [1], Charette [5], CharetteDrumm [8; 9], Charette-Drumm-Goldman-Morrill [11], Drumm [13], Goldman [20] and Drumm-Goldman [16; 18].

Let $\mathbf{E}_{1}^{3}$ denote Minkowski $(2+1)$-spacetime, that is, a simply connected complete three-dimensional flat Lorentzian manifold. Alternatively $\mathbf{E}_{1}^{3}$ is an affine space whose underlying vector space $\mathbb{R}_{1}^{3}$ of translations is a Lorentzian inner vector space, a vector space with an inner product

$$
\begin{aligned}
\mathbb{R}_{1}^{3} \times \mathbb{R}_{1}^{3} & \longrightarrow \mathbb{R} \\
(\mathrm{v}, \mathrm{w}) & \longmapsto \mathrm{v} \cdot \mathrm{w}
\end{aligned}
$$

of signature $(2,1)$.

A vector $x \in \mathbb{R}_{1}^{3}$ is

- $n$ ull if $\mathrm{x} \cdot \mathrm{x}=0$;

- timelike if $\mathrm{x} \cdot \mathrm{x}<0$;

- spacelike if $\mathrm{x} \cdot \mathrm{x}>0$.

A spacelike vector $\mathrm{x}$ is unit spacelike if $\mathrm{x} \cdot \mathrm{x}=1$. A null vector is future-pointing if its third coordinate is positive - this corresponds to choosing a connected component of the set of timelike vectors, or a time-orientation. 
Define the Lorentzian cross-product as follows. Choose an orientation on $\mathbb{R}_{1}^{3}$. Let

$$
\mathbb{R}_{1}^{3} \times \mathbb{R}_{1}^{3} \times \mathbb{R}_{1}^{3 \stackrel{\text { Det }}{\longrightarrow}} \mathbb{R}
$$

denote the standard determinant on the three-dimensional real vector space. The Lorentzian cross-product is the unique bilinear map

$$
\mathbb{R}_{1}^{3} \times \mathbb{R}_{1}^{3 \stackrel{\otimes}{\longrightarrow}} \mathbb{R}_{1}^{3}
$$

satisfying

$$
\mathrm{u} \cdot(\mathrm{v} \otimes \mathrm{w})=\operatorname{Det}(\mathrm{u}, \mathrm{v}, \mathrm{w}) .
$$

The following facts are well known (see for example Ratcliffe [31]):

Lemma 1.1 Let $u, v, x, y \in \mathbb{R}_{1}^{3}$. Then

$$
\begin{aligned}
u \cdot(x \otimes y) & =x \cdot(y \otimes u), \\
(u \otimes v) \cdot(x \otimes y) & =(u \cdot y)(v \cdot x)-(u \cdot x)(v \cdot y) .
\end{aligned}
$$

For a spacelike vector v, define its Lorentz-orthogonal plane to be

$$
\mathrm{v}^{\perp}=\{\mathrm{x} \mid \mathrm{x} \cdot \mathrm{v}=0\} .
$$

It is an indefinite plane, since the Lorentzian inner product restricts to an inner product of signature $(1,1)$. In particular, $v^{\perp}$ contains two null lines. The two future-pointing linearly independent vectors of Euclidean length 1 in this set are denoted $v^{-}$and $v^{+}$ and are chosen so that $\left(\mathrm{v}^{-}, \mathrm{v}^{+}, \mathrm{v}\right)$ is a positively oriented basis for $\mathbb{R}_{1}^{3}$.

Lemma 1.2 Let $v \in \mathbb{R}_{1}^{3}$ be a unit spacelike vector. Then

$$
\mathrm{v} \otimes \mathrm{v}^{+}=\mathrm{v}^{+} \text {and } \mathrm{v}^{-} \otimes \mathrm{v}=\mathrm{v}^{-} \text {. }
$$

The lemma follows immediately from Lemma 1.1 (see Charette-Drumm [9]).

Recall that $G$ is the group of all affine transformations that preserve the Lorentzian scalar product on the space of directions; $G$ is isomorphic to $O(2,1) \ltimes \mathbb{R}_{1}^{3}$. We shall restrict our attention to those transformations whose linear parts are in the identity component $\mathrm{SO}(2,1)^{0}$ of $\mathrm{O}(2,1)$, thus preserving orientation and time-orientation. As above, $L$ denotes the projection onto the linear part of an affine transformation.

Suppose $g \in \mathrm{SO}(2,1)^{0}$ and $g \neq \mathbb{I}$.

- $g$ is hyperbolic if it has three distinct real eigenvalues;

- $g$ is parabolic if its only eigenvalue is 1 ; 
- $g$ is elliptic if it has no real eigenvalues.

Denote the set of hyperbolic elements in $\mathrm{SO}(2,1)^{0}$ by $\mathrm{Hyp}_{0}$ and the set of parabolic elements by Par 0 .

We also call $\gamma \in \mathrm{G}$ hyperbolic (respectively parabolic, elliptic) if its linear part $\mathrm{L}(\gamma)$ is hyperbolic (respectively parabolic, elliptic). Denote the set of hyperbolic elements in $G$ by Hyp and the set of parabolic transformations by Par.

Let $\gamma \in \operatorname{Hyp} \cup \operatorname{Par}$. The eigenspace $\operatorname{Fix}(\mathrm{L}(\gamma))$ is one-dimensional. Let $v \in \operatorname{Fix}(\mathrm{L}(\gamma))$ be a non-zero vector and $x \in \mathbf{E}_{1}^{3}$. Define

$$
\tilde{\alpha}_{\mathrm{v}}(\gamma):=(\gamma(x)-x) \cdot \mathrm{v}
$$

The following facts are proved in Abels [1], Charette-Drumm [8; 9], Goldman [20], Drumm-Goldman [18] and Goldman-Margulis [23]:

- $\tilde{\alpha}_{\mathrm{v}}(\gamma)$ is independent of $x$;

- $\tilde{\alpha}_{\mathrm{v}}(\gamma)$ is identically zero if and only if $\gamma$ fixes a point;

- For any $\eta \in \mathrm{G}$,

$$
\tilde{\alpha}_{h(v)}\left(\eta \gamma \eta^{-1}\right)=\tilde{\alpha}_{\mathrm{v}}(\gamma)
$$

where $v \in \operatorname{Fix}(g)$ and $h=\mathrm{L}(\eta)$;

- For any $n \in \mathbb{Z}$,

$$
\tilde{\alpha}_{v}\left(\gamma^{n}\right)=|n| \tilde{\alpha}_{v}(\gamma)
$$

A linear transformation $g$ induces a natural orientation on $\operatorname{Fix}(g)$ as follows.

Definition 1.3 Let $g \in \mathrm{Hyp}_{0} \cup \operatorname{Par}_{0}$. A vector $\vee \in \operatorname{Fix}(g)$ is positive relative to $g$ if and only if

$$
(\mathrm{v}, \mathrm{x}, \mathrm{gx})
$$

is a positively oriented basis, where $\mathrm{x}$ is any null or timelike vector which is not an eigenvector of $g$.

The sign of $\gamma$ is the sign of $\tilde{\alpha}_{\mathrm{v}}(\gamma)$, where $\mathrm{v}$ is any positive vector in $\operatorname{Fix}(g)$. For $n<0$ the orientation of $\operatorname{Fix}\left(g^{n}\right)$ reverses, so $\gamma$ and $\gamma^{-1}$ have equal sign.

Lemma 1.4 (Margulis [27; 28], Charette-Drumm [8]) Let $\gamma_{1}, \gamma_{2} \in$ Hyp $\cup$ Par and suppose $\gamma_{1}$ and $\gamma_{2}$ have opposite signs. Then $\left\langle\gamma_{1}, \gamma_{2}\right\rangle$ does not act properly on $\mathbf{E}_{1}^{3}$. 
Let $\Gamma_{0} \subset \mathrm{O}(2,1)$ be a subgroup and $\rho$ an affine deformation of $\Gamma_{0}$,

$$
\rho(g)(x)=g(x)+u(g)
$$

where $x \in \mathbb{R}_{1}^{3}$. Then $\Gamma_{0} \stackrel{u}{\rightarrow} \mathbb{R}_{1}^{3}$ is a cocycle of $\Gamma_{0}$ with coefficients in the $\Gamma_{0}$-module $\mathbb{R}_{1}^{3}$ corresponding to the linear action of $\Gamma_{0}$. As affine deformations of $\Gamma_{0}$ correspond to cocycles in $Z^{1}\left(\Gamma_{0}, \mathbb{R}_{1}^{3}\right)$, translational equivalence classes of affine deformations comprise the cohomology group $\mathrm{H}^{1}\left(\Gamma_{0}, \mathbb{R}_{1}^{3}\right)$.

If $g \in \mathrm{Hyp}_{0}$, set $\mathrm{x}_{g}^{0}$ to be the unique positive vector in $\operatorname{Fix}(g)$ such that $\mathrm{x}_{g}^{0} \cdot \mathrm{x}_{g}^{0}=1$. If $g \in \operatorname{Par}_{0}$, choose a positive vector in $\operatorname{Fix}(g)$ and call it $x_{g}^{0}$.

Let $u \in \mathrm{Z}^{1}\left(\Gamma_{0}, \mathbb{R}_{1}^{3}\right)$. Reinterpreting the Margulis invariant as a linear functional on the space of cocycles $Z^{1}\left(\Gamma_{0}, \mathbb{R}_{1}^{3}\right)$, set

$$
\begin{aligned}
\Gamma_{0} \stackrel{\alpha_{[u]}}{\longrightarrow} \mathbb{R} \\
g \longmapsto \widetilde{\alpha}_{x_{g}^{0}}(\gamma),
\end{aligned}
$$

where $\gamma=\rho(g)$ is the affine deformation corresponding to $u(g)$. As the notation indicates, $\alpha_{[u]}$ only depends on the cohomology class of $u$, since $\widetilde{\alpha}_{x_{g}^{0}}$ is a class function.

\section{Hyperbolic geometry and the three-holed sphere}

Let $\Sigma$ denote a complete hyperbolic surface homeomorphic to a three-holed sphere. Each of the three ends can either flare out (that is, have infinite area) or end in a cusp. In the former case, a loop going around the end will have hyperbolic holonomy, and parabolic holonomy in the latter case. We consider certain geodesic laminations on the surface from which we will construct crooked fundamental polyhedra.

Fixing some arbitrary basepoint in $\Sigma$, let $\Gamma_{0}$ denote the image under the holonomy representation of the fundamental group of $\Sigma$. We may thus identify $\Sigma$ with $\mathbf{H}^{2} / \Gamma_{0}$. The fundamental group of $\Sigma$ is free of rank two and admits a presentation

$$
\Gamma_{0}=\left\langle g_{1}, g_{2}, g_{3} \mid g_{1} g_{2} g_{3}=\mathbb{I}\right\rangle,
$$

where the $g_{i}$ correspond to the components of $\partial \Sigma$ and may be hyperbolic or parabolic. For the rest of the paper, the $g_{i}$ and their affine deformations $\gamma_{i}$ are indexed by $i=1,2,3$ with addition in $\mathbb{Z} / 3 \mathbb{Z}$.

If $g_{i}$ is hyperbolic, it admits a unique invariant axis $l_{i} \subset \mathbf{H}^{2}$ which projects to an end of the three-holed sphere. For $g_{i}$ parabolic, we think of this invariant line as shrunk to 
a point on the ideal boundary. Since $\Gamma_{0}$ is discrete, the $l_{i}$ 's are pairwise disjoint, as in Figure 1.

For hyperbolic $g_{i}$, set $x_{i}^{+}, x_{i}^{-}$to be its attracting and repelling fixed points, respectively; if $g_{i}$ is parabolic, set $x_{i}^{+}=x_{i}^{-}$to be its unique fixed point.

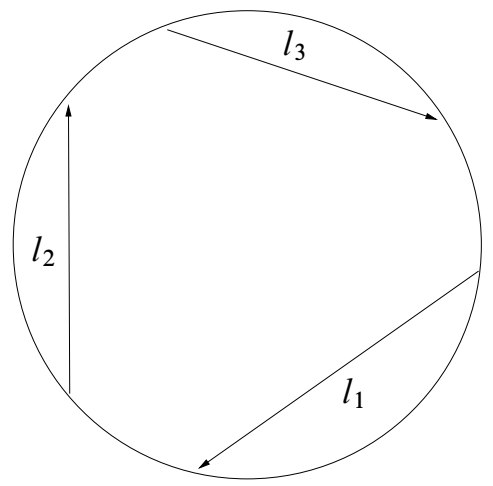

Figure 1: The invariant lines for $g_{1}, g_{2}, g_{3}$, with direction indicated by the arrows The three arcs in $\mathbf{H}^{2}$ respectively joining $\mathrm{x}_{i}^{+}$to $\mathrm{x}_{i-1}^{+}$project to a geodesic lamination of $\Sigma$ as drawn in Figures 2 and 3.
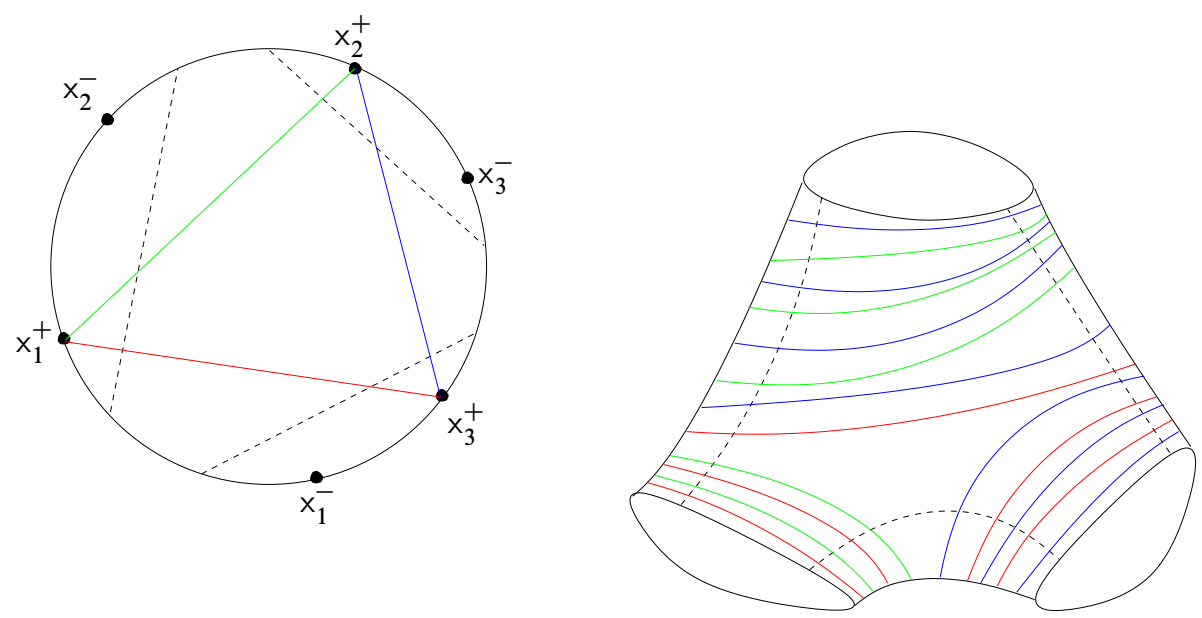

Figure 2: Three lines in $\mathbf{H}^{2}$ joining endpoints of the invariant axes $l_{i}$. On the right, the induced lamination of $\Sigma$.

We shall adapt the Kleinian model for $\mathbf{H}^{2}$ to the affine Lorentzian setting, as follows. A future-pointing timelike ray is a ray $q+\mathbb{R}_{+} \mathrm{w}$, where $q \in \mathbf{E}_{1}^{3}$ is a point and $\mathrm{w} \in \mathbb{R}_{1}^{3}$ is a future-pointing timelike vector. Parallelism defines an equivalence relation on 


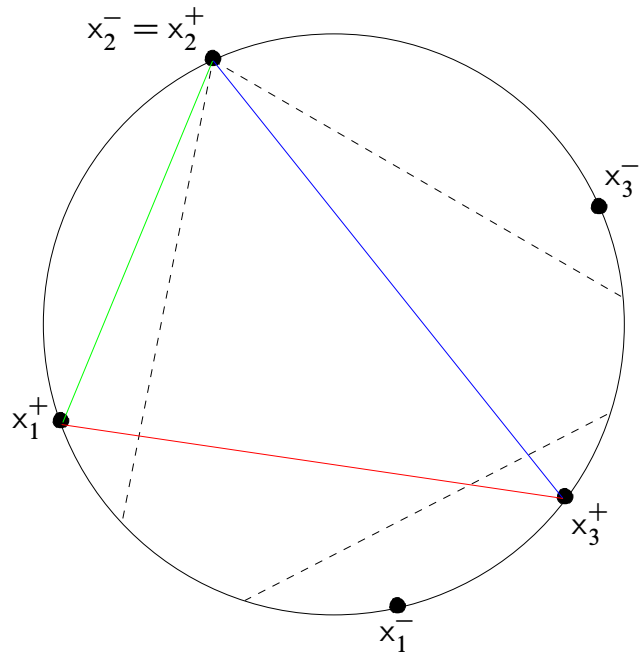

Figure 3: Three lines in $\mathbf{H}^{2}$ joining endpoints of $l_{i}$, with $g_{2}$ parabolic and $l_{2}$ an ideal point

future-pointing timelike rays, and points of $\mathbf{H}^{2}$ identify with equivalence classes of future-pointing timelike rays.

Denote by $\left[q+\mathbb{R}_{+} \mathrm{w}\right]$ the point in $\mathbf{H}^{2}$ corresponding to the equivalence class of the ray $q+\mathbb{R}_{+}$w.

Geodesics in $\mathbf{H}^{2}$ naturally identify with parallelism classes of indefinite affine planes. A point $\left[q+\mathbb{R}_{+} \mathrm{w}\right] \in \mathbf{H}^{2}$ is incident to a geodesic if and only if $\mathrm{w}$ is parallel to the geodesic's corresponding plane. A half-space $H$ in $\mathbf{E}_{1}^{3}$ bounded by an indefinite affine plane determines a half-plane $\mathfrak{H} \subset \mathbf{H}^{2}$. A point $\left[q+\mathbb{R}_{+}\right.$w] in $\mathbf{H}^{2}$ lies in $\mathfrak{H}$ if and only if $q+\mathbb{R}_{+} \mathrm{w}$ intersects $H$ in a ray, that is, $q+t \mathrm{w} \in H$ for $t \gg 0$.

Geodesics in $\mathbf{H}^{2}$ correspond dually to spacelike lines, since the Lorentz-perpendicular plane of a spacelike vector is indefinite. In the notation of (2), $\mathbb{R} x_{g_{i}}^{0}$ is dual to $l_{i}$ and the null vectors $\left(\mathrm{x}_{g_{i}}^{0}\right)^{ \pm}$respectively project to the ideal points $\mathrm{x}_{i}^{ \pm}$.

Furthermore spacelike vectors correspond to oriented geodesics, or equivalently to half-planes in $\mathbf{H}^{2}$. A spacelike vector spans a unique spacelike ray, which contains a unique unit spacelike vector $v$. The corresponding half-plane is

$$
\mathfrak{H}(\mathrm{v}):=\left\{\left[q+\mathbb{R}_{+} \mathrm{w}\right] \in \mathbf{H}^{2} \mid \mathrm{w} \cdot \mathrm{v} \geq 0\right\} .
$$

The geodesics $\partial \mathfrak{H}(\mathrm{u})$ and $\partial \mathfrak{H}(\mathrm{v}) \subset \mathbf{H}^{2}$ are respectively dual to $\mathrm{u}$ and $\mathrm{v}$. We distinguish three exclusive possibilities:

- $\quad \partial \mathfrak{H}(\mathrm{u})$ and $\partial \mathfrak{H}(\mathrm{v})$ intersect at a point inside $\mathbf{H}^{2}$ if $u \nabla v$ is timelike; 
- $\quad \partial \mathfrak{H}(u)$ and $\partial \mathfrak{H}(v)$ share an endpoint on $\partial \mathbf{H}^{2}$ if $u \nabla v$ is lightlike;

- $\quad \partial \mathfrak{H}(u)$ and $\partial \mathfrak{H}(v)$ neither intersect nor share an endpoint if $u \otimes v$ is spacelike.

Extending terminology from $\mathbf{H}^{2}$ to $\mathbb{R}_{1}^{3}$, say that two spacelike vectors $u, v \in \mathbb{R}_{1}^{3}$ are

- ultraparallel if $u \otimes v$ is spacelike,

- asymptotic if $\mathrm{u} \otimes \mathrm{v}$ is null, and

- crossing if $u \otimes v$ is lightlike.

\section{Crooked planes and half-spaces}

Crooked planes are Lorentzian analogs of equidistant surfaces. We will associate a triple of crooked planes to the lamination introduced in the previous section. We will see how to get pairwise disjoint crooked plane triples, yielding proper affine deformations of the linear holonomy. In this section, we define crooked planes.

Here is a somewhat technical, yet important, point. What we call crooked planes and half-spaces should really be called positively oriented crooked planes and half-spaces. We require crooked planes to be positively oriented when the signs of the Margulis invariants are positive. But for the case of negative Margulis invariants, we must use negatively oriented crooked planes. The arguments are essentially the same up to a change in sign - the curious reader should consult Drumm-Goldman [17].

Given a null vector $x \in \mathbb{R}_{1}^{3}$, set $\mathcal{P}(x)$ to be the set of (spacelike) vectors $w$ such that $\mathrm{w}^{+}$is parallel to $\mathrm{x}$. This half-plane in the Lorentz-orthogonal plane $\mathrm{x}^{\perp}$ is a connected component of $x^{\perp} \backslash\langle x\rangle$. If $v$ is a spacelike vector, then

$$
v \in \mathcal{P}\left(\mathrm{v}^{+}\right) \quad \text { and } \quad-v \in \mathcal{P}\left(\mathrm{v}^{-}\right) \text {. }
$$

Let $p \in \mathbf{E}_{1}^{3}$ be a point and $v \in \mathbb{R}_{1}^{3}$ a spacelike vector. Define the crooked plane $\mathcal{C}(v, p) \subset \mathbf{E}_{1}^{3}$ with vertex $p$ and direction vector $v$ to be the union of two wings

$$
p+\mathcal{P}\left(\mathrm{v}^{+}\right) \quad \text { and } \quad p+\mathcal{P}\left(\mathrm{v}^{-}\right)
$$

and a stem

$$
p+\left\{\mathrm{x} \in \mathbb{R}_{1}^{3} \mid \mathrm{v} \cdot \mathrm{x}=0, \mathrm{x} \cdot \mathrm{x} \leq 0\right\} .
$$

Each wing is a half-plane, and the stem is the union of two quadrants in an indefinite plane. The crooked plane itself is a piecewise linear submanifold, which stratifies into four connected open subsets of planes (two wings and the two components of the interior of the stem), four null rays, and a vertex. 
Definition 3.1 Let $\vee$ be a spacelike vector and $p \in \mathbf{E}_{1}^{3}$. The crooked half-space determined by $\vee$ and $p$, denoted $\mathrm{H}(\mathrm{v}, p)$, consists of all $q \in \mathbf{E}_{1}^{3}$ such that

- $(q-p) \cdot \mathrm{v}^{+} \leq 0$ if $(q-p) \cdot \mathrm{v} \geq 0$,

- $(q-p) \cdot \mathrm{v}^{-} \geq 0$ if $(q-p) \cdot \mathrm{v} \leq 0$, and

- either condition must hold for $q-p \in \mathrm{v}^{\perp}$.

Observe that $\mathcal{C}(\mathrm{v}, p)=\mathcal{C}(-\mathrm{v}, p)$. In contrast, the crooked half-spaces $\mathrm{H}(\mathrm{v}, p)$ and $\mathrm{H}(-\mathrm{v}, p)$ are distinct spaces. Their union and intersection are, respectively,

$$
\begin{aligned}
& \mathrm{H}(\mathrm{v}, p) \cup \mathrm{H}(-\mathrm{v}, p)=\mathbf{E}_{1}^{3} \\
& \mathrm{H}(\mathrm{v}, p) \cap \mathrm{H}(-\mathrm{v}, p)=\mathcal{C}(\mathrm{v}, p)=\mathcal{C}(-\mathrm{v}, p) .
\end{aligned}
$$

Crooked half-spaces in $\mathbf{E}_{1}^{3}$ determine half-planes in $\mathbf{H}^{2}$ as follows. As in the preceding section, a point in $\mathbf{H}^{2}$ corresponds to the equivalence class of a future-pointing timelike ray.

Lemma 3.2 Let $p, q \in \mathbf{E}_{1}^{3}$ and $\mathrm{v}, \mathrm{w} \in \mathbb{R}_{1}^{3}$ such that $\mathrm{v}$ is spacelike and $\mathrm{w}$ is timelike and future-pointing. Suppose that $\mathrm{H}(\mathrm{v}, p)$ is a crooked half-space and that $\mathrm{w} \cdot \mathrm{v} \neq 0$. Then $q+t \mathrm{w} \in \operatorname{int}(\mathrm{H}(\mathrm{v}, p))$ for $t \gg 0$ if and only if $[q+t \mathrm{w}] \in \operatorname{int}(\mathfrak{H}(\mathrm{v}))$.

Proof It suffices to consider the case that $p=0$ and

$$
v=\left[\begin{array}{l}
1 \\
0 \\
0
\end{array}\right]
$$

that is,

$$
\mathrm{H}(\mathrm{v}, p)=\left\{\left[\begin{array}{l}
x \\
y \\
z
\end{array}\right] \mid y+z \geq 0 \text { if } x \geq 0 \text { or } y-z \geq 0 \text { if } x \leq 0\right\} .
$$

By applying an automorphism preserving $\mathrm{H}(\mathrm{v}, p)$, we may assume

$$
q=\left[\begin{array}{l}
x_{0} \\
y_{0} \\
z_{0}
\end{array}\right], \quad \mathrm{w}=\left[\begin{array}{l}
d \\
0 \\
1
\end{array}\right] .
$$

where $|d|<1$.

Set $q(t):=q+t \mathrm{w}$. For any value of $d, q(t)$ eventually satisfies both $y+z=$ $y_{0}+z_{0}+t>0$ and $y-z<0$ for $t>0$.

Thus $q(t) \in \mathrm{H}(\mathrm{v}, p)$ if and only if $d>0$. On the other hand, the point $[q+t \mathrm{w}]$ lies in $\operatorname{int}(\mathfrak{H}(\mathrm{v}))$ if and only if $d>0$. The result follows. 


\section{Disjointness of crooked half-spaces}

This section discusses criteria for the disjointness of crooked half-spaces. Lemma 4.2 reduces disjointness of crooked half-spaces to disjointness of crooked planes. We need only consider pairs of crooked half-spaces in the case of ultraparallel or asymptotic vectors: when $\mathrm{u}$ and $\mathrm{v}$ are crossing $\mathcal{C}(\mathrm{u}, p)$ and $\mathcal{C}(\mathrm{v}, p)$ always intersect (see DrummGoldman [17]). Theorem 4.3 and Theorem 4.5 provide criteria for disjointness for crooked planes, and were established in [17]. Corollary 4.6 explicitly describes the set of disjoint crooked planes for a pair of asymptotic direction vectors.

Definition 4.1 Spacelike vectors $v_{1}, \ldots, v_{n} \in \mathbb{R}_{1}^{3}$ are consistently oriented if and only if, whenever $i \neq j$,

- $\mathrm{v}_{i} \cdot \mathrm{v}_{j}<0$;

- $\mathrm{v}_{i} \cdot \mathrm{v}_{j}^{ \pm} \leq 0$.

The second requirement implies that the $v_{i}$ are pairwise ultraparallel or asymptotic. Equivalently, $\mathrm{v}_{i}, \mathrm{v}_{j}, i \neq j$ are consistently oriented if and only if the interiors of the half-planes $\mathfrak{H}\left(\mathrm{v}_{i}\right)$ and $\mathfrak{H}\left(\mathrm{v}_{j}\right)$ are pairwise disjoint. (See Goldman [21, Section 4.2.1] for details.)

Lemma 4.2 Suppose $\mathrm{u}, \mathrm{v}$ are consistently oriented spacelike vectors, $p \in \mathbf{E}_{1}^{3}$, and $\mathrm{w}$ is a vector such that $\mathcal{C}(\mathrm{u}, p)$ and $\mathcal{C}(\mathrm{v}, p+\mathrm{w})$ are disjoint. Then $\mathcal{C}(\mathrm{v}, p+\mathrm{w}) \subset \mathrm{H}(-\mathrm{u}, p)$.

\section{Proof Because}

$$
\mathbf{E}_{1}^{3} \backslash \mathcal{C}(\mathrm{v}, p)=\operatorname{int}(\mathrm{H}(\mathrm{u}, p)) \cup \operatorname{int}(\mathrm{H}(-\mathrm{u}, p)),
$$

either $\mathcal{C}(\mathrm{v}, p+\mathrm{w}) \subset \mathrm{H}(\mathrm{u}, p)$ or $\mathcal{C}(\mathrm{v}, p+\mathrm{w}) \subset \mathrm{H}(-\mathrm{u}, p)$.

Suppose that $\mathcal{C}(\mathrm{v}, p+\mathrm{w}) \subset \mathrm{H}(\mathrm{u}, p)$. The future-pointing timelike rays on $\mathcal{C}(\mathrm{v}, p+\mathrm{w})$ lie on the stem of $\mathcal{C}(\mathrm{v}, p+\mathrm{w})$ and correspond to the geodesic $\partial \mathfrak{H}(\mathrm{v})$.

Since a future-pointing timelike ray on $\mathcal{C}(\mathrm{v}, p+\mathrm{w})$ lies entirely in $\mathrm{H}(\mathrm{u}, p)$, Lemma 3.2 implies that

$$
\partial \mathfrak{H}(\mathrm{v}) \subset \mathfrak{H}(\mathrm{u}) .
$$

Since $u, v$ are consistently oriented, the half-spaces $\mathfrak{H}(u)$ and $\mathfrak{H}(v)$ are disjoint, and $\mathfrak{H}(\mathrm{v}) \subset \mathfrak{H}(-\mathrm{u})$, a contradiction. Thus $\mathcal{C}(\mathrm{v}, p+\mathrm{w}) \subset \mathrm{H}(-\mathrm{u}, p)$ as desired.

Theorem 4.3 Let $v_{1}$ and $v_{2}$ be consistently oriented, ultraparallel, unit spacelike vectors and $p_{1}, p_{2} \in \mathbf{E}_{1}^{3}$. The crooked planes $\mathcal{C}\left(\mathrm{v}_{1}, p_{1}\right)$ and $\mathcal{C}\left(\mathrm{v}_{2}, p_{2}\right)$ are disjoint if and only if

$$
\left(p_{2}-p_{1}\right) \cdot\left(\mathrm{v}_{1} \otimes \mathrm{v}_{2}\right)>\left|\left(p_{2}-p_{1}\right) \cdot \mathrm{v}_{2}\right|+\left|\left(p_{2}-p_{1}\right) \cdot \mathrm{v}_{1}\right| .
$$


Corollary 4.4 Let $v_{1}, v_{2} \in \mathbb{R}_{1}^{3}$ be consistently oriented, ultraparallel vectors. Suppose

$$
p_{i}=a_{i} \mathrm{v}^{-}{ }^{-}-b_{i} \mathrm{v}_{i}{ }^{+},
$$

for $a_{i}, b_{i}>0, i=1,2$. Then $\mathcal{C}\left(\mathrm{v}_{1}, p_{1}\right)$ and $\mathcal{C}\left(\mathrm{v}_{2}, p_{2}\right)$ are disjoint.

Proof Rescaling if necessary, assume that $\mathrm{v}_{1}, \mathrm{v}_{2}$ are unit spacelike. Lemmas 1.1 and 1.2 imply that for $i \neq j$

$$
\begin{aligned}
\mathrm{v}_{i}{ }^{+} \cdot\left(\mathrm{v}_{i} \otimes \mathrm{v}_{j}\right) & =\mathrm{v}_{i}{ }^{+} \cdot \mathrm{v}_{j} \\
\mathrm{v}_{i}{ }^{-} \cdot\left(\mathrm{v}_{i} \otimes \mathrm{v}_{j}\right) & =-\mathrm{v}_{i}{ }^{-} \cdot \mathrm{v}_{j} .
\end{aligned}
$$

Consequently

$$
\begin{aligned}
\left(p_{2}-p_{1}\right) \cdot\left(\mathrm{v}_{1} \otimes \mathrm{v}_{2}\right) & =-\left(a_{2} \mathrm{v}_{2}^{-}+b_{2} \mathrm{v}_{2}^{+}\right) \cdot \mathrm{v}_{1}-\left(a_{1} \mathrm{v}_{1}{ }^{-}+b_{1} \mathrm{v}_{1}^{+}\right) \cdot \mathrm{v}_{2} \\
& =-a_{2} \mathrm{v}_{2}{ }^{-} \cdot \mathrm{v}_{1}-b_{2} \mathrm{v}_{2}{ }^{+} \cdot \mathrm{v}_{1}-a_{1} \mathrm{v}_{1}{ }^{-} \cdot \mathrm{v}_{2}-b_{1} \mathrm{v}_{1}^{+} \cdot \mathrm{v}_{2} \\
& >\left|\left(a_{2} \mathrm{v}_{2}{ }^{-}-b_{2} \mathrm{v}_{2}{ }^{+}\right) \cdot \mathrm{v}_{1}\right|+\left|\left(a_{1} \mathrm{v}_{1}{ }^{-}-b_{1} \mathrm{v}_{1}{ }^{+}\right) \cdot \mathrm{v}_{2}\right| .
\end{aligned}
$$

The above inequality follows because each term in the previous expression is positive since $v_{1}, v_{2}$ are consistently oriented. Finally

$$
\begin{aligned}
\left|\left(p_{2}-p_{1}\right) \cdot \mathrm{v}_{2}\right| & =\left|\left(a_{1} \mathrm{v}_{1}{ }^{-}-b_{1} \mathrm{v}_{1}{ }^{+}\right) \cdot \mathrm{v}_{2}\right| \\
\text { and } \quad\left|\left(p_{2}-p_{1}\right) \cdot \mathrm{v}_{1}\right| & =\left|\left(a_{2} \mathrm{v}_{2}{ }^{-}-b_{2} \mathrm{v}_{2}{ }^{+}\right) \cdot \mathrm{v}_{1}\right|,
\end{aligned}
$$

which completes the proof.

Alternatively, $\mathcal{C}\left(\mathrm{v}_{1}, p_{1}\right)$ and $\mathcal{C}\left(\mathrm{v}_{2}, p_{2}\right)$ are disjoint if and only if $p_{2}-p_{1}$ lies in the cone spanned by the four vectors

$$
\mathrm{v}_{2}^{-},-\mathrm{v}_{2}^{+},-\mathrm{v}_{1}^{-}, \mathrm{v}_{1}^{+} \text {. }
$$

Theorem 4.5 Let $v_{1}$ and $v_{2}$ be consistently oriented, asymptotic vectors such that $\mathrm{v}_{1}{ }^{-}=\mathrm{v}_{2}{ }^{+}$, and $p_{1}, p_{2} \in \mathbf{E}_{1}^{3}$. The crooked planes $\mathcal{C}\left(\mathrm{v}_{1}, p_{1}\right)$ and $\mathcal{C}\left(\mathrm{v}_{2}, p_{2}\right)$ are disjoint if and only if

$$
\begin{aligned}
& \left(p_{2}-p_{1}\right) \cdot \mathrm{v}_{1}<0, \\
& \left(p_{2}-p_{1}\right) \cdot \mathrm{v}_{2}<0, \\
& \left(p_{2}-p_{1}\right) \cdot\left(\mathrm{v}_{1}{ }^{+} \otimes \mathrm{v}_{2}{ }^{-}\right)>0 .
\end{aligned}
$$

If any of the above inequalities is an equality, the crooked planes intersect, but in such a way that $\mathcal{C}\left(\mathrm{v}_{1}, p_{1}\right) \subset \mathrm{H}\left(-\mathrm{v}_{2}, p_{2}\right)$ and vice versa. 
Corollary 4.6 Let $v_{1}, v_{2} \in \mathbb{R}_{1}^{3}$ be consistently oriented, asymptotic vectors such that $\mathrm{v}_{1}^{-}=\mathrm{v}_{2}{ }^{+}$. Suppose

$$
p_{i}=a_{i} \mathrm{v}^{-}{ }^{-}-b_{i} \mathrm{v}^{+}{ }^{+},
$$

where $a_{i}, b_{i}>0$ for $i=1,2$. Then $\mathcal{C}\left(\mathrm{v}_{1}, p_{1}\right)$ and $\mathcal{C}\left(\mathrm{v}_{2}, p_{2}\right)$ are disjoint.

\section{Proof Set}

$$
\mathrm{v}_{i}^{-} \otimes \mathrm{v}_{i}^{+}=\kappa_{i}^{2} \mathrm{v}_{i}
$$

for $i=1,2$. Then

$$
\begin{aligned}
& \left(p_{2}-p_{1}\right) \cdot \mathrm{v}_{1}=a_{2} \mathrm{v}_{2}^{-} \cdot \mathrm{v}_{1}<0 \\
& \left(p_{2}-p_{1}\right) \cdot \mathrm{v}_{2}=b_{1} \mathrm{v}_{1}{ }^{+} \cdot \mathrm{v}_{2}<0
\end{aligned}
$$

and

$$
\begin{aligned}
\left(p_{2}-p_{1}\right) \cdot\left(\mathrm{v}_{1}{ }^{+} \nabla \mathrm{v}_{2}{ }^{-}\right) & =-b_{2} \mathrm{v}_{2}^{+} \cdot\left(\mathrm{v}_{1}{ }^{+} \nabla \mathrm{v}_{2}{ }^{-}\right)-a_{1} \mathrm{v}_{2}{ }^{+} \cdot\left(\mathrm{v}_{1}{ }^{+} \nabla \mathrm{v}_{2}{ }^{-}\right) \\
& =-b_{2} \kappa_{2}^{2}\left(\mathrm{v}_{1}^{-} \cdot \mathrm{v}_{2}\right)-a_{1} \kappa_{2}^{2}\left(\mathrm{v}_{1}{ }^{+} \cdot \mathrm{v}_{2}\right) \\
& >0 .
\end{aligned}
$$

This completes the proof.

Keeping the notation in the statement in Corollary 4.6, we thus obtain disjoint crooked planes if and only if $p_{2}-p_{1}$ lies in a cone spanned by three vectors

$$
\mathrm{v}_{1}^{+}, \quad \mathrm{v}_{1}^{-}=\mathrm{v}_{2}^{+}, \quad \mathrm{v}_{2}^{-} \text {. }
$$

In (5), we allow $b_{2}=0$ or $a_{1}=0$. If $a_{2}=0, b_{1}=0$ or $a_{1}=b_{2}=0$, then the crooked planes intersect, but in a nice way, by the second part of Theorem 4.5.

\section{Crooked fundamental polyhedra}

Now look at how collections of pairwise disjoint crooked planes correspond to groups acting properly on $\mathbf{E}_{1}^{3}$. Let $v, v^{\prime} \in \mathbb{R}_{1}^{3}$ be two spacelike vectors. Suppose $\gamma \in \mathrm{G}$ and $p, p^{\prime} \in \mathbf{E}_{1}^{3}$ satisfy

$$
\gamma(\mathcal{C}(\mathrm{v}, p))=\mathcal{C}\left(\mathrm{v}^{\prime}, p^{\prime}\right)
$$

Then $\gamma(p)=p^{\prime}$ and $\mathrm{L}(\gamma)(\mathrm{v})$ is a scalar multiple of $\mathrm{v}^{\prime}$. In particular, $\gamma(\mathrm{H}(\mathrm{v}, p))$ is one of the two crooked half-spaces bounded by $\mathcal{C}\left(\mathrm{v}^{\prime}, p^{\prime}\right)$. 
Theorem 5.1 Suppose that $\mathrm{H}\left(\mathrm{v}_{i}, p_{i}\right)$ are $2 n$ pairwise disjoint crooked half-spaces and $\gamma_{1}, \ldots \gamma_{n} \in$ Hyp $\cup$ Par are such that for all $i$,

$$
\gamma_{i}\left(\mathrm{H}\left(\mathrm{v}_{-i}, p_{-i}\right)\right)=\mathbf{E}_{1}^{3} \backslash \operatorname{int}\left(\mathrm{H}\left(\mathrm{v}_{i}, p_{i}\right)\right) .
$$

Then $\Gamma=\left\langle\gamma_{1}, \ldots \gamma_{n}\right\rangle$ acts freely and properly on $\mathbf{E}_{1}^{3}$ with fundamental polyhedron

$$
\Omega=\mathbf{E}_{1}^{3} \backslash \bigcup_{-n \leq i \leq n} \operatorname{int}\left(\mathrm{H}\left(\mathrm{v}_{i}, p_{i}\right)\right) .
$$

Proof Here is an outline of the proof, given in Drumm [14; 15]. (See also CharetteGoldman [12].)

In order to show that $\bigcup_{\gamma \in \Gamma} \gamma(\Omega)=\mathbf{E}_{1}^{3}$, suppose on the contrary that $p \in \mathbf{E}_{1}^{3}$ is such that $p \notin \bigcup_{\gamma \in \Gamma} \gamma(\Omega)$. Then $p$ lies in a sequence of nested crooked half-spaces. Taking a $\Gamma$-translate of $p$ if necessary, we may assume that this sequence contains a subsequence of crooked half-spaces corresponding to hyperbolic elements of $\Gamma$.

However, hyperbolic elements in such a nested sequence are subject to a lower bound on the amount of compression they induce on a weak unstable plane. Consequently, the crooked planes bounding the crooked half-spaces lie a minimal distance away from each other, contradicting the existence of $p$.

Theorem 5.1 allows considerable flexibility in our choice of fundamental domains, in comparison to the standard construction (as in [15]). A crooked fundamental polyhedron $\Delta$ in $\mathbf{E}_{1}^{3}$ for $\Gamma$ determines a polygon $D$ in $\mathbf{H}^{2}$ for $L(\Gamma)$; the stems of $\partial \Delta$ define lines in $\mathbf{H}^{2}$ bounding D. However, while $\Gamma \cdot \Delta=\mathbf{E}_{1}^{3}$, the union $\mathrm{L}(\Gamma) \cdot \mathrm{D}$ may only be a proper open subset of $\mathbf{H}^{2}$. In the present case, this is the universal covering of the interior of the convex core of $\Sigma$. The convex core is an incomplete hyperbolic surface bounded by three closed geodesics. In contrast, the flat Lorentz manifold $\mathbf{E}_{1}^{3} / \Gamma$ is complete. While the hyperbolic fundamental polyhedra $L(\gamma)(D)$ only fill a proper subset of $\mathbf{H}^{2}$, the crooked fundamental polyhedra $\gamma(\Delta)$ fill all of $\mathbf{E}_{1}^{3}$.

Theorem 5.1 extends to the case when two of the crooked planes intersect in a single point.

Lemma 5.2 (Kissing Lemma) Let $\mathrm{u}_{1}, \mathrm{u}_{2}, \mathrm{v}_{1}, \mathrm{v}_{2} \in \mathbb{R}_{1}^{3}$ be pairwise consistently oriented vectors and suppose $q, p_{1}, p_{2} \in \mathbf{E}_{1}^{3}$ satisfy

$$
\begin{aligned}
\mathcal{C}\left(\mathrm{v}_{1}, p_{1}\right) \cap \mathcal{C}\left(\mathrm{v}_{2}, p_{2}\right) & =\varnothing \\
\mathcal{C}\left(\mathrm{v}_{1}, p_{1}\right) \cap \mathcal{C}\left(\mathrm{u}_{1}, q\right) & =\mathcal{C}\left(\mathrm{v}_{1}, p_{1}\right) \cap \mathcal{C}\left(\mathrm{u}_{2}, q\right)=\varnothing \\
\mathcal{C}\left(\mathrm{v}_{2}, p_{2}\right) \cap \mathcal{C}\left(\mathrm{u}_{1}, q\right) & =\mathcal{C}\left(\mathrm{v}_{1}, p_{1}\right) \cap \mathcal{C}\left(\mathrm{u}_{2}, q\right)=\varnothing
\end{aligned}
$$


Let $\gamma_{1}, \gamma_{2} \in \mathrm{G}$ such that $\gamma_{i}\left(\mathrm{H}\left(\mathrm{u}_{i}, q\right)\right)=\mathrm{H}\left(-\mathrm{v}_{i}, p_{i}\right)$. Then there exist $q_{1}, q_{2} \in \mathbf{E}_{1}^{3}$ such that the crooked planes

$$
\mathcal{C}\left(\mathrm{u}_{1}, q_{1}\right), \quad \mathcal{C}\left(\mathrm{u}_{2}, q_{2}\right), \quad \mathcal{C}\left(\mathrm{v}_{1}, \gamma_{1}\left(q_{1}\right)\right), \quad \mathcal{C}\left(\mathrm{v}_{2}, \gamma_{2}\left(q_{2}\right)\right)
$$

are pairwise disjoint.

Proof We will prove the lemma for the case where $u_{1}$ and $u_{2}$ are asymptotic, as it is the only case used in this paper. (The ultraparallel case is not much harder.)

Relabeling if necessary, assume that $\mathrm{u}_{1}{ }^{-}=\mathrm{u}_{2}{ }^{+}$. Let $\mathrm{v}_{0}$ be the unit spacelike vector that is a positive multiple of $\mathrm{u}_{2}^{-} \otimes \mathrm{u}_{1}{ }^{+}$. Corollaries 4.4, 4.6 imply that

$$
\mathcal{C}\left(\mathrm{v}_{0}, q\right) \cap \mathcal{C}\left(\mathrm{v}_{1}, p_{1}\right)=\mathcal{C}\left(\mathrm{v}_{0}, q\right) \cap \mathcal{C}\left(\mathrm{v}_{2}, p_{2}\right)=\varnothing .
$$

Thus by Lemma 3.2, $\mathrm{H}\left(\mathrm{v}_{0}, q\right)$ contains both $\mathrm{H}\left(\mathrm{u}_{1}, q\right)$ and $\mathrm{H}\left(\mathrm{u}_{2}, q\right)$ and furthermore, its complement contains $\mathcal{C}\left(\mathrm{v}_{1}, p_{1}\right)$ and $\mathcal{C}\left(\mathrm{v}_{2}, p_{2}\right)$. By openness of the disjointness conditions in Theorems 4.3 and 4.5 , there exist $\epsilon_{1}, \epsilon_{2} \in \mathbb{R}$ such that, for any $p_{i}^{\prime}$ in an $\epsilon_{i}$-neighborhood of $p_{i}, i=1,2$, the three crooked planes $\mathcal{C}\left(\mathrm{v}_{1}, p_{1}^{\prime}\right), \mathcal{C}\left(\mathrm{v}_{2}, p_{2}^{\prime}\right)$, $\mathcal{C}\left(v_{0}, q\right)$ remain disjoint.

Set

$$
q_{1}=\delta \mathrm{u}_{1}{ }^{-}-\delta^{\prime} \mathrm{u}_{1}{ }^{+}
$$

where $\delta, \delta^{\prime}>0$ are small enough so that $\gamma_{1}\left(q_{1}\right)$ lies in the $\epsilon_{1}$-neighborhood of $p_{1}$. Corollary 4.6 implies that $\mathcal{C}\left(\mathrm{u}_{1}, q_{1}\right) \cap \mathrm{H}\left(\mathrm{v}_{0}, q\right)=\varnothing$ and by Lemma $4.2, \mathcal{C}\left(\mathrm{u}_{1}, q_{1}\right) \subset$ $\mathrm{H}\left(\mathrm{v}_{0}, q\right)$.

Next, set $q_{2}=\delta^{\prime \prime} \mathrm{u}_{2}{ }^{-}$, where $\delta^{\prime \prime}>0$ is small enough so that $\gamma_{2}\left(q_{2}\right)$ lies in the $\epsilon_{2}$-neighborhood of $p_{2}$. Then $\mathcal{C}\left(\mathrm{u}_{2}, q_{2}\right) \subset \mathrm{H}\left(\mathrm{v}_{0}, q\right)$.

Finally, $q_{1}, q_{2}$ satisfy the condition in Corollary 4.6 and thus $\mathcal{C}\left(\mathrm{u}_{1}, q_{1}\right) \cap \mathcal{C}\left(\mathrm{u}_{2}, q_{2}\right)=\varnothing$.

\section{The space of proper affine deformations}

We parametrize the space of translational equivalence classes $\mathrm{H}^{1}\left(\Gamma_{0}, \mathbb{R}_{1}^{3}\right)$ of affine deformations of $\Gamma_{0}$ by Margulis invariants corresponding to $g_{1}, g_{2}, g_{3}$. Positivity of the three signs will guarantee a triple of crooked planes arising from the lamination described in Section 2. Alternatively, if the signs are all negative, use negatively oriented crooked planes (see Drumm-Goldman [17]) as mentioned in Section 3. The existence of such a crooked polyhedron thereby completes the proof of Theorem A.

We begin with the parametrization of $\mathrm{H}^{1}\left(\Gamma_{0}, \mathbb{R}_{1}^{3}\right)$. 
Lemma 6.1 Let $\pi$ denote a free group of rank two with presentation

$$
\left\langle A_{1}, A_{2}, A_{3} \mid A_{1} A_{2} A_{3}=\mathbb{I}\right\rangle .
$$

Let $\pi \stackrel{\rho_{0}}{\longrightarrow} \mathrm{SO}(2,1)^{0}$ be a homomorphism such that $\rho_{0}\left(A_{i}\right) \in \mathrm{Hyp}_{0} \cup \operatorname{Par}_{0}$ for $i=1,2,3$. Suppose that $\rho_{0}(\pi)$ is not solvable. For each $i$ choose a vector $\mathrm{x}_{i} \in \operatorname{Fix}\left(\rho_{0}\left(A_{i}\right)\right)$ positive with respect to $\rho_{0}\left(A_{i}\right)$ and define

$$
\begin{aligned}
\mathrm{H}^{1}\left(\Gamma_{0}, \mathbb{R}_{1}^{3}\right) \stackrel{\mu_{i}}{\longrightarrow} \mathbb{R} \\
{[u] \longmapsto \widetilde{\alpha}_{x_{i}}\left(\rho\left(A_{i}\right)\right)=u\left(A_{i}\right) \cdot \mathrm{x}_{i} }
\end{aligned}
$$

where $\rho$ is the affine deformation corresponding to $u$. Then

$$
\begin{aligned}
\mathrm{H}^{1}\left(\Gamma_{0}, \mathbb{R}_{1}^{3}\right) \stackrel{\mu}{\longrightarrow} \mathbb{R}^{3} \\
\mu:[u] \longmapsto\left[\begin{array}{l}
\mu_{1}([u]) \\
\mu_{2}([u]) \\
\mu_{3}([u])
\end{array}\right]
\end{aligned}
$$

is a linear isomorphism of vector spaces.

Of course, this lemma is much more general than our specific application. In our application $\rho_{0}$ is an isomorphism of $\pi=\pi_{1}(\Sigma)$ onto the discrete subgroup $\Gamma_{0} \subset$ $\mathrm{SO}(2,1)^{0}$, and corresponds to a complete hyperbolic three-holed sphere $\operatorname{int}(\Sigma)$. The generators $A_{1}, A_{2}, A_{3}$ correspond to the three components of $\partial \Sigma$.

The proof of Lemma 6.1 is postponed to Appendix A.

Conclusion of proof of Theorem A In the presentation of $\Gamma_{0}$

$$
\Gamma_{0}=\left\langle g_{1}, g_{2}, g_{3} \mid g_{1} g_{2} g_{3}=\mathbb{I}\right\rangle
$$

relabel, if necessary, to assume that the invariant axes are ordered as in Figures 1 and 4. As in Section 1, choose a positive vector $x_{i}^{0}:=x_{g_{i}}^{0} \in \operatorname{Fix}\left(g_{i}\right)$, further requiring that $x_{i}^{0}$ be unit spacelike when $g_{i}$ is hyperbolic. The assumption on the invariant axes implies that the vectors $x_{i}^{0}$ are pairwise consistently oriented. With this fixed choice of positive vectors

$$
\mu_{i}([u])=\alpha_{[u]}\left(g_{i}\right) .
$$

We will now show that every positive cocycle $\left(\mu_{1}, \mu_{2}, \mu_{3}\right) \in Z^{1}\left(\Gamma_{0}, \mathbb{R}_{1}^{3}\right)$ corresponds to a triple of mutually disjoint crooked planes arising from the geodesic lamination described in Section 2. 
By a slight abuse of notation, set $x_{i}^{+}=\left(x_{i}^{0}\right)^{+}$and $x_{i}^{+}=x_{i}^{0}$ when $g_{i}$ is parabolic. The three consistently oriented unit spacelike vectors

$$
\mathrm{v}_{i}=\frac{-1}{\mathrm{x}_{i}^{+} \cdot \mathrm{x}_{i+1}^{+}} \mathrm{x}_{i+1}^{+} \otimes \mathrm{x}_{i}^{+}
$$

correspond to the arcs joining $\mathrm{x}_{i+1}^{+}$to $\mathrm{x}_{i}^{+}$in $\mathbf{H}^{2}$.

For $i=1,2,3$, choose $a_{i}, b_{i}>0$. Then set

$$
p_{i}=a_{i} \mathrm{x}_{i+1}^{+}-b_{i} \mathrm{x}_{i}^{+} .
$$

By Corollary 4.6, the crooked planes $\mathcal{C}\left(\mathrm{v}_{i}, p_{i}\right)$ are pairwise disjoint, since $\mathrm{v}_{i}^{-}=\mathrm{x}_{i+1}^{+}$ and $\mathrm{v}_{i}^{+}=\mathrm{x}_{i}^{+}$.

Let $u$ be the cocycle such that, for $i=1,2,3$, the affine deformation $\gamma_{i}$ of $g_{i}$ satisfies

$$
\gamma_{i}\left(p_{i}\right)=p_{i-1}
$$

Then

$$
\begin{aligned}
\gamma_{1}^{-1}\left(\mathcal{C}\left(\mathrm{v}_{3}, p_{3}\right)\right) & =\mathcal{C}\left(g_{1}^{-1}\left(\mathrm{v}_{3}\right), p_{1}\right) \\
\gamma_{2}\left(\mathcal{C}\left(\mathrm{v}_{2}, p_{2}\right)\right) & =\mathcal{C}\left(g_{2}\left(\mathrm{v}_{2}\right), p_{1}\right) .
\end{aligned}
$$

Since $g_{1}^{-1}\left(\mathrm{x}_{3}^{+}\right)$is parallel to $g_{2}\left(\mathrm{x}_{3}^{+}\right)$, the vectors $g_{1}^{-1}\left(\mathrm{v}_{3}\right)$ and $g_{2}\left(\mathrm{v}_{2}\right)$ are ultraparallel. Furthermore

$$
\mathcal{C}\left(-g_{1}^{-1}\left(\mathrm{v}_{3}\right), p_{1}\right), \mathcal{C}\left(-g_{2}\left(\mathrm{v}_{2}\right), p_{1}\right) \subset \mathrm{H}\left(\mathrm{v}_{1}, p_{1}\right) .
$$

See Figure 4. We can thus apply the Kissing Lemma 5.2 to obtain a crooked fundamental polyhedron for the cocycle $u$, so that Theorem 5.1 holds.

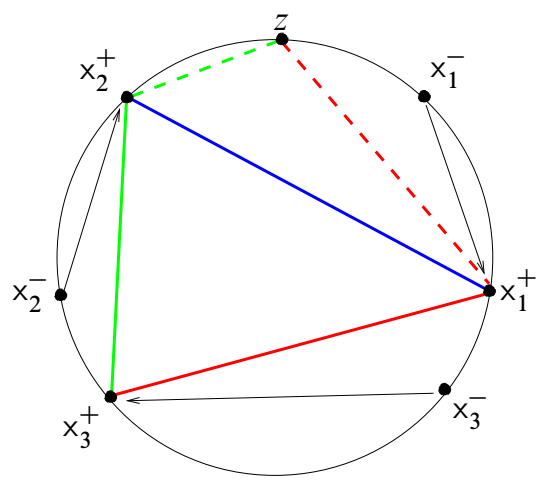

Figure 4: The template for a crooked fundamental polyhedron. Here $z=$ $g_{1}^{-1}\left(\mathrm{x}_{3}^{+}\right) \| g_{2}\left(\mathrm{x}_{3}^{+}\right)$. 
Every positive cocycle arises in this way. Indeed, compute the Margulis invariants for the cocycle $u$ :

$$
\begin{aligned}
\mu_{i} & =\left(p_{i-1}-p_{i}\right) \cdot \mathrm{x}_{i}^{0} \\
& =\left(a_{i-1} \mathrm{x}_{i}^{+}-b_{i-1} \mathrm{x}_{i-1}^{+}-a_{i} \mathrm{x}_{i+1}^{+}+b_{i} \mathrm{x}_{i}^{+}\right) \cdot \mathrm{x}_{i}^{0} \\
& =\left(-a_{i} \mathrm{x}_{i+1}^{+}-b_{i-1} \mathrm{x}_{i-1}^{+}\right) \cdot \mathrm{x}_{i}^{0}
\end{aligned}
$$

Every product $\beta_{i, j}=-\mathrm{x}_{i}^{+} \cdot \mathrm{x}_{j}^{0}$ is positive, because the vectors $\mathrm{x}_{j}^{0}$ are pairwise consistently oriented. In matrix form

$$
\left[\begin{array}{l}
\mu_{1} \\
\mu_{2} \\
\mu_{3}
\end{array}\right]=\left[\begin{array}{cccccc}
\beta_{2,1} & 0 & 0 & 0 & 0 & \beta_{3,1} \\
0 & \beta_{1,2} & \beta_{3,2} & 0 & 0 & 0 \\
0 & 0 & 0 & \beta_{2,3} & \beta_{1,3} & 0
\end{array}\right]\left[\begin{array}{l}
a_{1} \\
b_{1} \\
a_{2} \\
b_{2} \\
a_{3} \\
b_{3}
\end{array}\right]
$$

and every positive triple of values $\left(\mu_{1}, \mu_{2}, \mu_{3}\right)$ may be realized by choosing appropriate positive values of $a_{i}, b_{i}$. Explicitly, for $i=1,2,3$, choose $p_{i}, q_{i}>0$ with $p_{i}+q_{i}=1$, and define

$$
\left[\begin{array}{l}
a_{1} \\
b_{1} \\
a_{2} \\
b_{2} \\
a_{3} \\
b_{3}
\end{array}\right]=\left[\begin{array}{l}
p_{1} \mu_{1} / \beta_{2,1} \\
q_{2} \mu_{2} / \beta_{1,2} \\
p_{2} \mu_{2} / \beta_{3,2} \\
q_{3} \mu_{3} / \beta_{2,3} \\
p_{3} \mu_{3} / \beta_{1,3} \\
q_{1} \mu_{1} / \beta_{3,1}
\end{array}\right]
$$

The proof of Theorem A is complete.

\section{Embedding in an arithmetic group}

As an application, we construct examples of proper affine deformations of a Fuchsian group as subgroups of the symplectic group $\operatorname{Sp}(4, \mathbb{R})$.

Give $\mathbb{R}^{4}$ the symplectic form defined by the matrix

$$
\mathbb{J}:=\left[\begin{array}{cccc}
0 & 0 & 1 & 0 \\
0 & 0 & 0 & 1 \\
-1 & 0 & 0 & 0 \\
0 & -1 & 0 & 0
\end{array}\right] .
$$


Let $S p(4, \mathbb{R})$ denote the group of linear symplectomorphisms of $\mathbb{R}^{4}$ with this symplectic form.

Let $e_{1}, e_{2}, e_{3}, e_{4}$ denote the standard basis. Then the planes $L:=\left\langle e_{1}, e_{2}\right\rangle$ and $L^{\prime}:=$ $\left\langle e_{3}, e_{4}\right\rangle$ are Lagrangian with $\mathbb{R}^{4}=L \oplus L^{\prime}$. The symplectic form defines a dual pairing

$$
L \times L^{\prime} \longrightarrow \mathbb{R}
$$

and every linear automorphism $L \stackrel{g}{\rightarrow} L$ extends to a linear symplectomorphism $g \oplus$ $\left(g^{\dagger}\right)^{-1}$ of $\mathbb{R}^{4}$. Let $G_{0}$ denote the corresponding embedding $\mathrm{GL}(2, \mathbb{R}) \hookrightarrow \operatorname{Sp}(4, \mathbb{R})$.

Let $S_{2}$ be the vector space of $2 \times 2$ symmetric matrices, with a Lorentzian inner product defined by the negative of the determinant. This vector group embeds in $\operatorname{Sp}(4, \mathbb{R})$ as the unipotent subgroup $U$ consisting of all block matrices

$$
\left[\begin{array}{ll}
\mathbb{I} & S \\
0 & \mathbb{I}
\end{array}\right]
$$

where $S \in \mathrm{S}_{2}$. This subgroup comprises all linear symplectomorphisms of $\mathbb{R}^{4}$ which act identically both on $L$ and on the quotient space $\mathbb{R}^{4} / L$. A model for Minkowski space $\mathbf{E}_{1}^{3}$ is the set $A$ of all Lagrangian planes in $\mathbb{R}^{4}$ which are transverse to $L$. The unipotent group $U$ acts simply transitively on $A$, and we regard this as the group of translations of $\mathbf{E}_{1}^{3}$. Under the identification of $A$ with $\mathbf{E}_{1}^{3}$, the subgroup generated by $U$ and $G_{0}$ acts as the group of orientation-preserving isometries. $G_{0}$ corresponds to the subgroup of linear isometries, where $L^{\prime}$ corresponds to the origin.

We construct subgroups of $\operatorname{Sp}(4, \mathbb{Z})$ which act properly on the $S_{2}$ model of $\mathbf{E}_{1}^{3}$. The linear parts and translational parts of Lorentzian transformations of $S_{2}$ are associated with elements of $\operatorname{Sp}(4, \mathbb{Z})$. The level two congruence subgroup $\Gamma_{0}$ of $\operatorname{SL}(2, \mathbb{Z})$ is generated by

$$
g_{1}:=-\left[\begin{array}{ll}
1 & 2 \\
0 & 1
\end{array}\right], g_{2}:=-\left[\begin{array}{cc}
1 & 0 \\
-2 & 1
\end{array}\right], g_{3}:=\left[\begin{array}{ll}
1 & -2 \\
2 & -3
\end{array}\right] .
$$

subject to the relation $g_{1} g_{2} g_{3}=\mathbb{I}$. It is freely generated by $g_{1}$ and $g_{2}$. All three $g_{i}$ are parabolic and the quotient hyperbolic surface $\Sigma=\mathbf{H}^{2} / \Gamma_{0}$ is a three-punctured sphere. The symmetric matrices

$$
\mathrm{x}_{1}:=\left[\begin{array}{cc}
-1 & 0 \\
0 & 0
\end{array}\right], \quad \mathrm{x}_{2}:=\left[\begin{array}{cc}
0 & 0 \\
0 & -1
\end{array}\right], \quad \mathrm{x}_{3}:=\left[\begin{array}{cc}
-1 & -1 \\
-1 & -1
\end{array}\right]
$$

define positive fixed vectors with respect to $g_{1}, g_{2}$ and $g_{3}$. The triple $\left(\mathrm{x}_{1}, \mathrm{x}_{2}, \mathrm{x}_{3}\right)$ defines a decoration of $\Sigma$. 
An affine deformation of $\Gamma_{0}$ is defined by two arbitrary vectors $u_{1}, u_{2} \in \mathrm{S}_{2}$ as translational parts

$$
u_{1}:=\left[\begin{array}{ll}
a_{1} & b_{1} \\
b_{1} & c_{1}
\end{array}\right] \quad \text { and } \quad u_{2}:=\left[\begin{array}{ll}
a_{2} & b_{2} \\
b_{2} & c_{2}
\end{array}\right]
$$

Thus the affine transformations with linear part $g_{i}$ and translational part $u_{i}$ are

$$
\begin{aligned}
\gamma_{1}: & =\left[\begin{array}{cccc}
1 & 0 & a_{1} & b_{1} \\
0 & 1 & b_{1} & c_{1} \\
0 & 0 & 1 & 0 \\
0 & 0 & 0 & 1
\end{array}\right]\left[\begin{array}{cccc}
-1 & -2 & 0 & 0 \\
0 & -1 & 0 & 0 \\
0 & 0 & -1 & 0 \\
0 & 0 & 2 & -1
\end{array}\right] \\
\text { and } & \gamma_{2}:=\left[\begin{array}{llll}
1 & 0 & a_{2} & b_{2} \\
0 & 1 & b_{2} & c_{2} \\
0 & 0 & 1 & 0 \\
0 & 0 & 0 & 1
\end{array}\right]\left[\begin{array}{cccc}
-1 & 0 & 0 & 0 \\
2 & -1 & 0 & 0 \\
0 & 0 & -1 & -2 \\
0 & 0 & 0 & -1
\end{array}\right] .
\end{aligned}
$$

The corresponding Margulis invariants, taken with respect to $\mathrm{x}_{1}, \mathrm{x}_{2}, \mathrm{x}_{3}$, are

$$
\begin{aligned}
& \mu_{1}=c_{1}, \\
& \mu_{2}=a_{2}, \\
& \mu_{3}=c_{1}+c_{2}-2 b_{1}+2 b_{2}+a_{1}+a_{2} .
\end{aligned}
$$

By Theorem A, the affine deformation $\Gamma:=\left\langle\gamma_{1}, \gamma_{2}\right\rangle$ acts properly with crooked fundamental polyhedron whenever

$$
\begin{aligned}
& \mu_{1}>0 \\
& \mu_{2}>0 \\
& \mu_{3}>0 .
\end{aligned}
$$

Furthermore, taking $a_{1}, b_{1}, c_{1}, a_{2}, b_{2}, c_{2} \in \mathbb{Z}$ implies $\Gamma \subset \mathrm{Sp}(4, \mathbb{Z})$.

Here are some explicit examples. Consider the slice for translational equivalence defined by $b_{1}=b_{2}=c_{2}=0$. Choose three positive integers $\mu_{1}, \mu_{2}, \mu_{3}$. Take

$$
\begin{aligned}
a_{1} & =\mu_{3}-\mu_{1}-\mu_{2} \\
c_{1} & =\mu_{1} \\
a_{2} & =\mu_{2},
\end{aligned}
$$

that is, let

$$
\gamma_{1}:=\left[\begin{array}{cccc}
1 & 0 & \mu_{3}-\mu_{1}-\mu_{2} & 0 \\
0 & 1 & 0 & \mu_{1} \\
0 & 0 & 1 & 0 \\
0 & 0 & 0 & 1
\end{array}\right]\left[\begin{array}{cccc}
-1 & -2 & 0 & 0 \\
0 & -1 & 0 & 0 \\
0 & 0 & -1 & 0 \\
0 & 0 & 2 & -1
\end{array}\right]
$$


and

$$
\gamma_{2}:=\left[\begin{array}{cccc}
1 & 0 & \mu_{2} & 0 \\
0 & 1 & 0 & 0 \\
0 & 0 & 1 & 0 \\
0 & 0 & 0 & 1
\end{array}\right]\left[\begin{array}{cccc}
-1 & 0 & 0 & 0 \\
2 & -1 & 0 & 0 \\
0 & 0 & -1 & -2 \\
0 & 0 & 0 & -1
\end{array}\right]
$$

\section{Appendix A Proof of Lemma 6.1}

Recall the statement of the lemma:

Lemma Let $\pi$ denote a free group of rank two with presentation

$$
\left\langle A_{1}, A_{2}, A_{3} \mid A_{1} A_{2} A_{3}=\mathbb{I}\right\rangle .
$$

Let $\pi \stackrel{\rho_{0}}{\longrightarrow} \mathrm{SO}(2,1)^{0}$ be a homomorphism such that $\rho_{0}\left(A_{i}\right) \in \mathrm{Hyp}_{0} \cup \mathrm{Par}_{0}$ for $i=1,2,3$. Suppose that $\rho_{0}(\pi)$ is not solvable. For each $i$ choose a vector $\mathrm{x}_{i} \in \operatorname{Fix}\left(\rho_{0}\left(A_{i}\right)\right)$ positive with respect to $\rho_{0}\left(A_{i}\right)$ and let

$$
\begin{aligned}
\mathrm{H}^{1}\left(\Gamma_{0}, \mathbb{R}_{1}^{3}\right) \stackrel{\mu_{i}}{\longrightarrow} \mathbb{R} \\
{[u] \longmapsto \widetilde{\alpha}_{x_{i}}\left(\rho\left(A_{i}\right)\right)=u\left(A_{i}\right) \cdot \mathrm{x}_{i} }
\end{aligned}
$$

where $\rho$ is the affine deformation corresponding to $u$. Then

$$
\mu:[u] \longmapsto\left[\begin{array}{l}
\mu_{1}([u]) \\
\mu_{2}([u]) \\
\mu_{3}([u])
\end{array}\right]
$$

is an isomorphism between the vector spaces $\mathrm{H}^{1}\left(\Gamma_{0}, \mathbb{R}_{1}^{3}\right)$ and $\mathbb{R}^{3}$.

Proof First lift $\rho_{0}$ to a representation $\pi \stackrel{\tilde{\rho}_{0}}{\longrightarrow} \mathrm{SL}(2, \mathbb{R})$ under the double covering $\mathrm{SL}(2, \mathbb{R}) \longmapsto \mathrm{SO}(2,1)^{0}$. The condition that $\rho_{0}(\pi)$ is not solvable implies that the representation $\tilde{\rho}_{0}$ on $\mathbb{R}^{2}$ is irreducible. By a well-known classic theorem (see, for example, Goldman [21]), such a representation is determined up to conjugacy by the three traces

$$
a_{i}:=\operatorname{tr}\left(\tilde{\rho}_{0}\left(A_{i}\right)\right) .
$$

and, choosing $b_{3}$ such that $b_{3}+1 / b_{3}=a_{3}$, we may conjugate $\tilde{\rho}_{0}$ to the representation defined by

(6) $\tilde{\rho}_{0}\left(A_{1}\right)=\left[\begin{array}{cc}a_{1} & -1 \\ 1 & 0\end{array}\right], \quad \tilde{\rho}_{0}\left(A_{2}\right)=\left[\begin{array}{cc}0 & -b_{3} \\ 1 / b_{3} & a_{2}\end{array}\right], \quad \tilde{\rho}_{0}\left(A_{2}\right)=\left[\begin{array}{cc}b_{3} & -a_{1} c_{3}+a_{2} \\ 0 & 1 / b_{3}\end{array}\right]$. 
Since $\pi$ is freely generated by $A_{1}, A_{2}$, a cocycle $\pi \stackrel{u}{\rightarrow} \mathbb{R}_{1}^{3}$ is completely determined by two values $u\left(A_{1}\right), u\left(A_{2}\right) \in \mathbb{R}_{1}^{3}$. Furthermore, since $\rho_{0}(\pi)$ is nonsolvable, the coboundary map

$$
\mathbb{R}_{1}^{3} \stackrel{\partial}{\rightarrow} Z^{1}\left(\Gamma_{0}, \mathbb{R}_{1}^{3}\right)
$$

is injective. Therefore the vector space $\mathrm{H}^{1}\left(\Gamma_{0}, \mathbb{R}_{1}^{3}\right)$ has dimension three.

To show that the linear map $\mu$ is an isomorphism, it suffices to show that $\mu$ is onto. To this end, it suffices to show that for each $i=1,2,3$ there is a cocycle $u \in \mathrm{Z}^{1}\left(\Gamma_{0}, \mathbb{R}_{1}^{3}\right)$ such that $u\left(A_{i}\right) \neq 0$ and $u\left(A_{j}\right)=0$ for $j \neq i$. By cyclic symmetry it is only necessary to do this for $i=1$.

Under the local isomorphism $\mathrm{SL}(2, \mathbb{R}) \longmapsto \mathrm{SO}(2,1)^{0}$, the Lie algebra $\mathfrak{s l}(2, \mathbb{R})$ maps to the Lie algebra $\mathfrak{s o}(2,1)$ which in turn maps isomorphically to the Lorentzian vector space $\mathbb{R}_{1}^{3}$. (Compare Goldman-Margulis [23], Goldman [20] and Charette-DrummGoldman [10].) If $g \in \mathrm{SL}(2, \mathbb{R})$ is hyperbolic or parabolic, then a neutral eigenvector $\mathrm{x}_{g}^{0}$ is a nonzero multiple of the traceless projection

$$
\widehat{g}:=g-\frac{\operatorname{tr}(g)}{2} \mathbb{I} .
$$

Define a cocycle for the representation $\tilde{\rho}_{0}$ defined in (6) by

$$
u\left(A_{1}\right):=\left[\begin{array}{ll}
1 & 0 \\
0 & 0
\end{array}\right], \quad u\left(A_{2}\right):=\left[\begin{array}{ll}
0 & 0 \\
0 & 0
\end{array}\right], \quad u\left(A_{3}\right):=\left[\begin{array}{cc}
0 & 0 \\
-1 / c & 0
\end{array}\right] .
$$

Then $\mu_{1}(u) \neq 0$ but $\mu_{2}(u)=\mu_{3}(u)=0$ as claimed. The proof of Lemma 6.1 is complete.

\section{References}

[1] H Abels, Properly discontinuous groups of affine transformations: a survey, Geom. Dedicata 87 (2001) 309-333 MR1866854

[2] I Agol, Tameness of hyperbolic 3-manifolds arXiv:math.GT/0405568

[3] T Barbot, V Charette, T Drumm, W M Goldman, K Melnick, A primer on the (2+ 1) Einstein universe, from: "Recent developments in pseudo-Riemannian geometry", ESI Lect. Math. Phys., Eur. Math. Soc., Zürich (2008) 179-229 MR2436232

[4] D Calegari, D Gabai, Shrinkwrapping and the taming of hyperbolic 3-manifolds, J. Amer. Math. Soc. 19 (2006) 385-446 MR2188131

[5] V Charette, Affine deformations of ultraideal triangle groups, Geom. Dedicata 97 (2003) 17-31 MR2003687 Special volume dedicated to the memory of Hanna Miriam Sandler (1960-1999) 
[6] V Charette, The affine deformation space of a rank two Schottky group: a picture gallery, Geom. Dedicata 122 (2006) 173-183 MR2295549

[7] V Charette, Non-proper affine actions of the holonomy group of a punctured torus, Forum Math. 18 (2006) 121-135 MR2206247

[8] V Charette, T Drumm, Strong marked isospectrality of affine Lorentzian groups, J. Differential Geom. 66 (2004) 437-452 MR2106472

[9] V Charette, T A Drumm, The Margulis invariant for parabolic transformations, Proc. Amer. Math. Soc. 133 (2005) 2439-2447 MR2138887

[10] V Charette, T A Drumm, W Goldman, Stretching three-holed spheres and the Margulis invariant, from: "In the tradition of Ahlfors-Bers V", Contemp. Math. 510, Amer. Math. Soc., Providence, RI (2010) 61-70 MR2581843

[11] V Charette, T Drumm, W Goldman, M Morrill, Complete flat affine and Lorentzian manifolds, Geom. Dedicata 97 (2003) 187-198 MR2003697 Special volume dedicated to the memory of Hanna Miriam Sandler (1960-1999)

[12] V Charette, W M Goldman, Affine Schottky groups and crooked tilings, from: "Crystallographic groups and their generalizations (Kortrijk, 1999)", Contemp. Math. 262, Amer. Math. Soc., Providence, RI (2000) 69-97 MR1796126

[13] T A Drumm, Examples of nonproper affine actions, Michigan Math. J. 39 (1992) 435-442 MR1182499

[14] T A Drumm, Fundamental polyhedra for Margulis space-times, Topology 31 (1992) 677-683 MR1191372

[15] T A Drumm, Linear holonomy of Margulis space-times, J. Differential Geom. 38 (1993) 679-690 MR1243791

[16] T A Drumm, W M Goldman, Complete flat Lorentz 3-manifolds with free fundamental group, Internat. J. Math. 1 (1990) 149-161 MR1060633

[17] T A Drumm, W M Goldman, The geometry of crooked planes, Topology 38 (1999) 323-351 MR1660333

[18] T A Drumm, W M Goldman, Isospectrality of flat Lorentz 3-manifolds, J. Differential Geom. 58 (2001) 457-465 MR1906782

[19] D Fried, W M Goldman, Three-dimensional affine crystallographic groups, Adv. in Math. 47 (1983) 1-49 MR689763

[20] W M Goldman, The Margulis invariant of isometric actions on Minkowski $(2+1)-$ space, from: "Rigidity in dynamics and geometry (Cambridge, 2000)", Springer, Berlin (2002) 187-201 MR1919401

[21] W M Goldman, Trace coordinates on Fricke spaces of some simple hyperbolic surfaces, from: "Handbook of Teichmüller theory Vol II", IRMA Lect. Math. Theor. Phys. 13, Eur. Math. Soc., Zürich (2009) 611-684 MR2497777 
[22] W M Goldman, F Labourie, G Margulis, Proper affine actions and geodesic flows of hyperbolic surfaces, Ann. Math. 170 (2009) 1051-1083

[23] W M Goldman, G A Margulis, Flat Lorentz 3-manifolds and cocompact Fuchsian groups, from: "Crystallographic groups and their generalizations (Kortrijk, 1999)", Contemp. Math. 262, Amer. Math. Soc., Providence, RI (2000) 135-145 MR1796129

[24] W M Goldman, G A Margulis, Y Minsky, Complete flat Lorentz 3-manifolds and laminations of hyperbolic surfaces, in preparation

[25] C Jones, Pyramids of properness, PhD thesis, University of Maryland (2003)

[26] F Labourie, Fuchsian affine actions of surface groups, J. Differential Geom. 59 (2001) 15-31 MR1909247

[27] G A Margulis, Free completely discontinuous groups of affine transformations, Dokl. Akad. Nauk SSSR 272 (1983) 785-788 MR722330

[28] G A Margulis, Complete affine locally flat manifolds with a free fundamental group, Zap. Nauchn. Sem. Leningrad. Otdel. Mat. Inst. Steklov. (LOMI) 134 (1984) 190-205 MR741860 Automorphic functions and number theory II

[29] G Mess, Lorentz spacetimes of constant curvature, Geom. Dedicata 126 (2007) 3-45 MR2328921

[30] J Milnor, On fundamental groups of complete affinely flat manifolds, Advances in Math. 25 (1977) 178-187 MR0454886

[31] J G Ratcliffe, Foundations of hyperbolic manifolds, second edition, Graduate Texts in Mathematics 149, Springer, New York (2006) MR2249478

Département de mathématiques, Université de Sherbrooke

Sherbrooke, Québec J1K 2R1, Canada

Department of Mathematics, Howard University

Washington DC 20059, USA

Department of Mathematics, University of Maryland

College Park MD 20742, USA

v.charette@usherbrooke.ca, tdrumm@howard.edu, wmg@math.umd.edu

Proposed: Walter Neumann

Seconded: Jean-Pierre Otal, Benson Farb
Received: 7 October 2009

Revised: 10 May 2010 